\title{
Young diagrams and intersection numbers for toric manifolds associated with Weyl chambers
}

\author{
Hiraku Abe \\ Osaka City University Advanced Mathematical Institute \\ 3-3-138 Sugimoto, Sumiyoshi-ku \\ Osaka 558-8585, Japan \\ hirakuabe@globe.ocn.ne.jp
}

Submitted: Apr 22, 2014; Accepted: Mar 20, 2015; Published: Apr 14, 2015

Mathematics Subject Classifications: 14M25, 17B22, 13F55

\begin{abstract}
We study intersection numbers of invariant divisors in the toric manifold associated with the fan determined by the collection of Weyl chambers for each root system of classical type and of exceptional type $G_{2}$. We give a combinatorial formula for intersection numbers of certain subvarieties which are naturally indexed by elements of the Weyl group. These numbers describe the ring structure of the cohomology of the toric manifold.
\end{abstract}

Keywords: Young diagrams; intersection numbers; toric varieties; structure constants

\section{Introduction}

Let $\Phi$ be a root system in the $n$-dimensional Euclidean space $E$ with its inner product. We denote by $\Delta(\Phi)$ the fan determined by the collection of Weyl chambers in $E^{*}$, and consider the toric manifold $X$ associated with $\Delta(\Phi)$. This toric manifold arises as the closure of a general orbit in the flag variety with respect to the standard torus action which makes $X$ a regular semisimple Hessenberg variety ([4]). The Weyl group $W$ naturally acts on the Weyl chambers and hence also on $X$. The representation of $W$ on the cohomology $H^{*}(X ; \mathbb{C})$ has been extensively studied by Procesi [9], Dolgachev-Lunts [5], and Stembridge [10]. For the classical root system of type $A_{n}$, Losev-Manin [8] described $X$ as the moduli space of stable $(n+1)$-pointed chains of projective lines (cf. Batyrev-Blume [1]).

Let $\Pi=\left\{\alpha_{1}, \cdots, \alpha_{n}\right\} \subset \Phi$ be a set of simple roots, then we have a torus invariant non-singular subvariety $X_{u}$ in $X$ of codimension $\left|u(\Pi) \cap \Phi^{-}\right|$such that the associated cohomology classes $\left\{\left[X_{u}\right]\right\}_{u \in W}$ form a module basis of the integral singular cohomology $H^{*}(X)$. The cohomology class $\left[X_{u}\right]$ is written as a monomial of torus invariant divisors 
$D_{u \omega_{i}}$ of $X$ for all coweights $u \omega_{i}$ satisfying $u \alpha_{i} \in \Phi^{-}$where $\left\{\omega_{1}, \cdots, \omega_{n}\right\}$ is the set of fundamental coweights (see Section 2 and (17) for details). In this paper, we study the case for the root systems of classical type and of exceptional type $G_{2}$, and we give a combinatorial formula of the intersection numbers

$$
\left(\mu_{X},\left[w_{0} X_{w_{0} w}\right]\left[X_{u}\right]\left[X_{v}\right]\right)
$$

of three subvarieties $X_{u}, X_{v}$ and $w_{0} X_{w_{0} w}$ for $u, v, w \in W$ where $\mu_{X}$ is the fundamental homology class of $X$ and $w_{0}$ is the longest element. As an application, we will obtain a recursive formula for the structure constants $c_{u, v}^{w}$ in the expansion of the product

$$
\left[X_{u}\right]\left[X_{v}\right]=\sum_{w \in W} c_{u, v}^{w}\left[X_{w}\right] \quad \text { where } \quad c_{u, v}^{w} \in \mathbb{Z}
$$

as discussed in Section 4.

Let us state our formula for $\left(\mu_{X},\left[w_{0} X_{w_{0} w}\right]\left[X_{u}\right]\left[X_{v}\right]\right)$ in the case of the classical root system of type $A_{n}$ (the results for the classical root systems of type $B_{n}, C_{n}$, and $D_{n}$ are stated in Section 5). In this case, the Weyl group $W$ is the $(n+1)$-th permutation group $\mathfrak{S}_{n+1}$. For each $u \in \mathfrak{S}_{n+1}$, we let

$$
\begin{aligned}
& D(u):=\{\{u(1), u(2), \cdots, u(i)\} \mid u(i)>u(i+1)\}, \\
& A(u):=\{\{u(1), u(2), \cdots, u(i)\} \mid u(i)<u(i+1)\}
\end{aligned}
$$

where each $\{u(1), u(2), \cdots, u(i)\}$ is a subset of $[n+1]$. We define a Young diagram $\lambda_{u v}^{w}$ as follows. Assume $d(u)+d(v)=d(w)$, and the collection $D(u) \coprod D(v) \coprod A(w)$ forms a nested chain of subsets of $[n+1]$. In this case, $\lambda_{u v}^{w}$ is defined to be the Young diagram consisting of the cardinalities of those chains ordered as a weakly decreasing sequence. Otherwise, $\lambda_{u v}^{w}=\emptyset$. For example, suppose $n=4$ and, let $u=12354, v=31254$, and $w=35421$. Then, we have that $D(u)=\{1235\}, D(v)=\{3,3125\}=\{3,1235\}$, and $A(w)=\{3\}$ where 1235 denotes the set $\{1,2,3,5\}$ and we use the same notation for others. These sets forms a nested chain of subsets $3 \subset 3 \subset 1235 \subset 1235$, and hence we obtain $\lambda_{12354,31254}^{35421}=(4,4,1,1)$.

For a Young diagram $\lambda=\left(\lambda_{1} \geqslant \cdots \geqslant \lambda_{n}\right)$ with $n$ rows (i.e. $\left.\lambda_{n}>0\right)$ fitting into the $n \times n$ square, we define $I(\lambda) \in \mathbb{Z}$ to be the following integer. Let $s$ be the number of lower-right corners of $\lambda$, i.e., $s=\left|\left\{i \in[n] \mid \lambda_{i}>\lambda_{i+1}\right\}\right|$ where $\lambda_{n+1}:=0$. Write

$$
\left\{i \in[n] \mid \lambda_{i}>\lambda_{i+1}\right\}=\left\{i_{1}, \cdots, i_{s}\right\} .
$$

We impose the condition $i_{1}<i_{2}<\cdots<i_{s}$ to determine them uniquely. Observe that $i_{s}=n$. For example, if $n=4$ and $\lambda=(4,2,2,1)$, then $s=3$ and $\left\{i_{1}, i_{2}, i_{3}\right\}=\{1,3,4\}$. For $r=1, \cdots, s$, define

$$
a_{r}:=i_{r}-i_{r-1}-1, \quad b_{r}:=\lambda_{i_{r}}-\lambda_{i_{r+1}}-1, \quad c_{r}:=\lambda_{i_{r}}+i_{r}-n-1
$$


where we write $i_{0}=0$, and let

$$
y_{r}:=\left(\begin{array}{l}
a_{r} \\
c_{r}
\end{array}\right)\left(\begin{array}{l}
b_{r} \\
c_{r}
\end{array}\right) \text { for } r=1, \cdots, s .
$$

We use the convention $\left(\begin{array}{l}x \\ y\end{array}\right)=0$ unless $0 \leqslant y \leqslant x$. By shading each lower-right corner of $\lambda$, the pictorial meanings of $a_{r}, b_{r}$, and $c_{r}$ become clear (as shown in Figure 1). Namely, $a_{r}$ is the number of boxes between the north side of the shaded box and the the upper-left corner placed above, $b_{r}$ is the similar number for the horizontal segment of the corner, and $c_{r}$ is the number of boxes between the north side of the shaded box and the crossing point of the vertical segment and the anti-diagonal line where we count negatively if that part of the vertical segment is above the anti-diagonal.

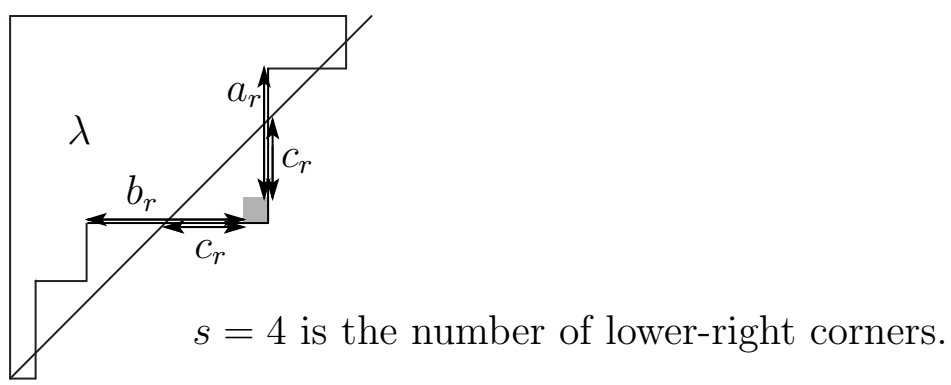

Figure 1: Three numbers $a_{r}, b_{r}$ and $c_{r}$

Now, let

$$
I(\lambda):=(-1)^{n+s} y_{1} \cdots y_{s},
$$

and put $I(\emptyset)=0$. The following is our main statement for type $A_{n}$.

Theorem 1. $\left(\mu_{X},\left[w_{0} X_{w_{0} w}\right]\left[X_{u}\right]\left[X_{v}\right]\right)=I\left(\lambda_{u v}^{w}\right)$ for any $u, v, w \in \mathfrak{S}_{n+1}$ where $\mu_{X}$ is the fundamental homology class of $X$.

We will prove Theorem 1 by computing general intersection numbers of invariant divisors of $X$ in Section 3 and 4. Section 5 is devoted to the classical root systems of type $B_{n}, C_{n}$ and $D_{n}$.

\section{Preliminaries}

Let $\Phi$ be a root system in the $n$-dimensional Euclidean space $E$ with its inner product. Let $M \subset E$ be the root lattice of $\Phi$ and $N \subset E^{*}$ be the coweight lattice of $\Phi$. Then $M$ is the dual lattice of $N$ with respect to the natural pairing.

We choose a set of simple roots $\Pi=\left\{\alpha_{1}, \cdots, \alpha_{n}\right\} \subset \Phi$, and let $\Pi^{*}:=\left\{\omega_{1}, \cdots, \omega_{n}\right\} \subset$ $E^{*}$ be the dual basis of $\Pi$ defined by $\left\langle\omega_{i}, \alpha_{j}\right\rangle=\delta_{i j}$, i.e., $\omega_{1}, \cdots, \omega_{n}$ are the fundamental coweights. For each $u \in W$, denote

$$
\sigma_{u}:=\operatorname{cone}\left(u \omega_{1}, \cdots, u \omega_{n}\right)=\left\{\sum_{i=1}^{n} \lambda_{i} u \omega_{i} \mid \lambda_{i} \geqslant 0\right\} .
$$


These cones $\left\{\sigma_{u}\right\}_{u \in W}$ form a non-singular complete fan $\Delta(\Phi)$ in $E^{*}$ by including all their faces. The set of minimal generators of these cones are the set of coweights:

$$
\Phi^{*}=\bigcup_{v \in W}\left\{v \omega_{1}, \cdots, v \omega_{n}\right\} .
$$

For each element $u \in W$, the maximal cones containing a minimal generator $u \omega_{i}$ are $\sigma_{v}$ for $v \in W$ such that $u \omega_{i}=v \omega_{j}$ for some $j$. There is a cone of $\Delta(\Phi)$ generated by minimal generators $x_{1}, \cdots, x_{k} \in \Phi^{*}$ if and only if there exists $u \in W$ such that each $x_{i}$ can be written as $u \omega_{j}$ for some $j$.

We consider the toric manifold $X=X(\Phi)$ associated with the fan $\Delta(\Phi)$. For root systems $\Phi$ and $\Phi^{\prime}$, it is easily verified that $X(\Phi) \cong X\left(\Phi^{\prime}\right)$ as toric varieties (in the sense of [3] Sec. 3.3.) if and only if $\Phi \cong \Phi^{\prime}$ as root systems (in the sense that their Cartan matrices are the same up to permuting the indexes). We refer to [1] and [7] for general properties of $X$. Let $D_{x} \subset X$ be the invariant divisor corresponding to the ray generated by $x \in \Phi^{*}$. The Poincaré dual $\tau_{x}:=\left[D_{x}\right]$ gives us a cohomology class of degree 2 in the integral singular cohomology $H^{*}(X)$. The cohomology ring $H^{*}(X)$ is isomorphic to the face ring of the underlying simplicial complex of the fan $\Delta(\Phi)$ modulo some linear relations ([6]). More precisely, we have

$$
H^{*}(X)=\mathbb{Z}\left[\tau_{x} \mid x \in \Phi^{*}\right] / I
$$

where the ideal $I$ is generated by $\tau_{x_{1}} \cdots \tau_{x_{k}}$ for which $x_{1}, \cdots, x_{k}$ do not generate a face of $\sigma_{u}$ for some $u \in W$ and $\sum_{x \in \Phi^{*}}\langle x, \alpha\rangle \tau_{x}$ for any root $\alpha$. Namely, we have the following equalities in $H^{*}(X)$ :

$$
\sum_{x \in \Phi^{*}}\langle x, \alpha\rangle \tau_{x}=0 \quad \text { for any root } \alpha
$$

The above observation about rays of $\Delta(\Phi)$ implies that

Lemma 2. We have $\tau_{x_{1}} \cdots \tau_{x_{k}}=0$ unless there exists $u \in W$ such that each $x_{i}$ can be written as $u \omega_{j}$ for some $j$.

Let $\mu_{X}$ be the fundamental homology class of $X$. For subvarieties $Z_{1}, \cdots, Z_{k} \subset X$, we call $\left(\mu_{X},\left[Z_{1}\right] \cdots\left[Z_{k}\right]\right)$ the intersection number of $Z_{1}, \cdots, Z_{k}$ where $\left[Z_{i}\right]$ denotes the Poincaré dual of $Z_{i}$. Note that the Weyl group $W$ acts on the fan $\Delta(\Phi)$, and hence acts on the toric manifold $X$. We have $u X_{x}=X_{u x}$ for any $u \in W$ and $x \in \Phi^{*}$ which means that $\left(u^{-1}\right)^{*} \tau_{x}=\tau_{u x}$. The next lemma says that intersection numbers for divisors $D_{u \omega_{i}}$ are invariant under the Weyl group action.

Lemma 3. Let $x_{1}, \cdots, x_{n} \in \Phi^{*}$. Then for any $u \in W$, we have

$$
\left(\mu_{X}, \tau_{u x_{1}} \cdots \tau_{u x_{n}}\right)=\left(\mu_{X}, \tau_{x_{1}} \cdots \tau_{x_{n}}\right) .
$$

Proof. Observe that $\tau_{u x_{1}} \cdots \tau_{u x_{n}}=\left(u^{-1}\right)^{*}\left(\tau_{x_{1}} \cdots \tau_{x_{n}}\right)$. Both of $\tau_{u x_{1}} \cdots \tau_{u x_{n}}$ and $\tau_{x_{1}} \cdots \tau_{x_{n}}$ can be written as the cohomology class $[p]$ of a point $p$ in $X$ multiplied by some integer, and these integers are the corresponding intersection numbers. Since $u$ preserves the orientation of $X$, we have $\left(u^{-1}\right)^{*}([p])=[u \cdot p]=[p]$ which proves the claim. 
For any $u \in W$, the product $\tau_{u \omega_{1}} \cdots \tau_{u \omega_{n}}$ is the Poincaré dual of a point in $X$ since the invariant divisors $X_{u \omega_{1}}, \cdots, X_{u \omega_{n}}$ intersect transversally which means that

$$
\left(\mu_{X}, \tau_{u \omega_{1}} \cdots \tau_{u \omega_{n}}\right)=1
$$

We will compute the intersection number $\left(\mu_{X}, \tau_{x_{1}} \cdots \tau_{x_{n}}\right)$ for arbitrary $x_{1}, \cdots, x_{n} \in \Phi^{*}$ for the root systems of classical type. By Lemma 2, we can assume that this number is of the form $\left(\mu_{X},\left(\tau_{u \omega_{i_{1}}}\right)^{m_{1}} \cdots\left(\tau_{u \omega_{i_{s}}}\right)^{m_{s}}\right)$ for some $1 \leqslant i_{1}<\cdots<i_{s} \leqslant n$ and $1 \leqslant m_{k} \leqslant n(k=$ $1, \cdots, s)$ satisfying $m_{1}+\cdots+m_{s}=n$ without loss of generality. We call the number $m_{k}$ the multiplicity of $\tau_{u \omega_{i_{k}}}$. We compute this number by applying the linear relations (3) to reduce the multiplicities $m_{1}, \cdots, m_{s}$. Although Lemma 3 shows that this number is equal to $\left(\mu_{X},\left(\tau_{\omega_{i_{1}}}\right)^{m_{1}} \cdots\left(\tau_{\omega_{i_{s}}}\right)^{m_{s}}\right)$, we will need Lemma 3 again after applying the relations (3).

In the next section, we will consider the classical root system of type $A_{n}$, and compute the intersection numbers.

\section{Intersection numbers for Type $A_{n}$}

In this section, we compute the intersection numbers for the toric manifold $X$ of type $A_{n}$. Let $E=\left\{x \in \mathbb{R}^{n+1} \mid x_{1}+\cdots+x_{n+1}=0\right\}$. The roots are $t_{i}-t_{j} \in E(1 \leqslant i, j \leqslant n+1)$ where $t_{i} \in \mathbb{R}^{n+1}$ is the $i$-th standard vector. We choose $\Pi=\left\{t_{i}-t_{i+1} \mid 1 \leqslant i \leqslant n\right\}$ as the set of simple roots, and write $\alpha_{i}=t_{i}-t_{i+1}$ for each $i$. The Weyl group $W=\mathfrak{S}_{n+1}$ is the $(n+1)$-th permutation group acting on $E$ by $u\left(t_{i}-t_{j}\right)=t_{u(i)}-t_{u(j)}$ for each $u \in W$. The minimal generators $\omega_{1}, \cdots, \omega_{n} \in E^{*}$ of the fundamental Weyl chamber $\sigma_{\text {id }}$ are

$$
\omega_{i}=\left(e_{1}+\cdots+e_{i}\right)-\frac{i}{n+1}\left(e_{1}+\cdots+e_{n+1}\right) \quad \text { for } \quad i=1, \cdots, n
$$

where $\left\{e_{i}\right\}_{i} \subset\left(\mathbb{R}^{n+1}\right)^{*}$ is the dual basis of $\left\{t_{i}\right\}_{i} \subset \mathbb{R}^{n+1}$.

Denoting by $2^{[n+1]}$ the set of all subsets of $[n+1]=\{1, \cdots, n+1\}$, we have a welldefined map $\Phi^{*} \rightarrow 2^{[n+1]}$ by sending $u \omega_{i} \mapsto\{u(1), \cdots, u(i)\}$. It is easy to see that this is an injection, and this establishes an identification

$$
\Phi^{*} \longleftrightarrow \quad \text { the set of non-empty proper subsets of }[n+1]
$$

In particular, the well-definedness implies that if $u \omega_{i}=v \omega_{j}$ then $i=j$. Now, for each $\emptyset \subsetneq S \subsetneq[n+1]$, we define $\tau_{S}:=\tau_{u \omega_{i}}$ where $u \omega_{i} \in \Phi^{*}$ corresponds to $S$ by this identification. Then, for $\emptyset \subsetneq S_{1}, \cdots, S_{q} \subsetneq[n+1](1 \leqslant q \leqslant n)$, it follows by Lemma 2 that $\tau_{S_{1}} \cdots \tau_{S_{q}}=0$ unless these sets form a nested chain of subsets, i.e. $S_{1} \subset \cdots \subset S_{q}$ up to reordering.

With Lemma 3, it is easy to show the following invariance property of intersection numbers which implies that $\left(\mu_{X}, \tau_{S_{1}} \cdots \tau_{S_{n}}\right)$ for $\emptyset \subsetneq S_{1} \subset \cdots \subset S_{n} \subsetneq[n+1]$ is determined by the set of integers $1 \leqslant\left|S_{1}\right| \leqslant \cdots \leqslant\left|S_{n}\right| \leqslant n$.

Lemma 4. Let $\emptyset \subsetneq S_{1} \subset \cdots \subset S_{n} \subsetneq[n+1]$ and $\emptyset \subsetneq S_{1}^{\prime} \subset \cdots \subset S_{n}^{\prime} \subsetneq[n+1]$. If $\left|S_{i}\right|=\left|S_{i}^{\prime}\right|$ for all $i=1, \cdots, n$, then $\left(\mu_{X}, \tau_{S_{1}} \cdots \tau_{S_{n}}\right)=\left(\mu_{X}, \tau_{S_{1}^{\prime}} \cdots \tau_{S_{n}^{\prime}}\right)$. 
Motivated by this property, we compute intersection numbers in terms of Young diagrams consisting of the cardinalities of the sets corresponding to the given invariant divisors. The linear relations (3) are translated to

$$
\sum_{\substack{\emptyset \subseteq S \subsetneq[n+1] \\ k \in S, l \notin S}} \tau_{S}-\sum_{\substack{\emptyset \subseteq S \subsetneq[n+1] \\ k \notin S, l \in S}} \tau_{S}=0 \quad \text { for each } k, l \in[n+1] .
$$

In the following, we write $\tau_{\emptyset}=\tau_{[n+1]}=1$. This equality together with the above observation about $\tau_{S_{1}} \cdots \tau_{S_{q}}$ being 0 implies the next lemma.

Lemma 5. Let $\emptyset \subset A \subsetneq B \subsetneq C \subset[n+1]$. For any $b \in B \backslash A$ and $c \in C \backslash B$, we have

$$
\tau_{A} \tau_{B}^{2} \tau_{C}=-\sum_{\substack{A \subsetneq B^{\prime} \subsetneq C \\ B^{\prime} \neq B, b \in B^{\prime}, c \notin B^{\prime}}} \tau_{A} \tau_{B} \tau_{B^{\prime}} \tau_{C} .
$$

For a Young diagram fitting into the $n \times n$ square, we write the dotted anti-diagonal line shifted down half the length of a single box from the standard anti-diagonal. (See Figure 2).
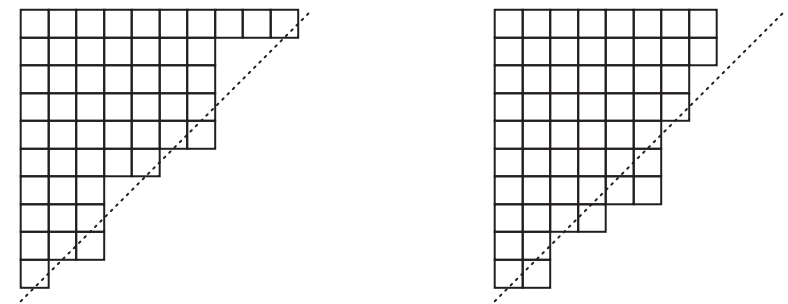

Figure 2: Young diagrams and the dotted anti-diagonal line

Let $\emptyset \subsetneq S_{1} \subset \cdots \subset S_{n} \subsetneq[n+1]$, and denote by $\lambda$ the Young diagram consisting of $\lambda_{i}=\left|S_{n+1-i}\right|$ for each $i$. We write $\lambda_{n+1}=0$. Let $s$ be the number of the lower-right corners of $\lambda$, that is,

$$
s:=\left|\left\{i \in[n] \mid \lambda_{i}>\lambda_{i+1}\right\}\right|
$$

Proposition 6. (Vanishing property) $\left(\mu_{X}, \tau_{S_{1}} \cdots \tau_{S_{n}}\right)=0$ unless each step of the zigzag line of the lower-right corners of $\lambda$ crosses the dotted anti-diagonal.

Proof. We suppose that there is a step of the zigzag line of $\lambda$ which does not cross the dotted anti-diagonal, and show $\left(\mu_{X}, \tau_{S_{1}} \cdots \tau_{S_{n}}\right)=0$ by induction on $k:=n-s$. Since there is no such case for $k=0$, we consider the case $k=1$. In this case, there is a unique vertical segment of length 2 in the zigzag line of $\lambda$. If there is a (unique) horizontal segment of length 2, then the vertical and horizontal segments are not adjacent because of our assumption. By applying Lemma 5 for the square corresponding to this vertical segment, it follows that the intersection number is zero since there is no summand. 
For the general case, take a vertical segment of length $\geqslant 2$. Let us say that this vertical segment contains $S_{i}$ and $S_{i+1}$ (i.e. $S_{i}=S_{i+1}$ ). We separate this square in $\tau_{S_{1}} \cdots \tau_{S_{n}}$ by Lemma 5. Let $\lambda^{\prime}$ be the Young diagram corresponding to a summand in the right-handside. Then the zigzag line of $\lambda^{\prime}$ has a step which does not cross the dotted anti-diagonal. In fact, if the vertical segment does not cross the dotted anti-diagonal, then this segment survives as a non-crossing segment of length at least 1, and if it does then we can find another vertical segment which does not, and this segment is preserved for each $\lambda^{\prime}$ in the summands. Now the induction hypothesis shows that each term will vanish after taking the intersection number, and we get $\left(\mu_{X}, \tau_{S_{1}} \cdots \tau_{S_{n}}\right)=0$.

Let $\lambda=\left(\lambda_{1} \geqslant \cdots \geqslant \lambda_{n}\right)$ be a Young diagram with $n$ rows (i.e. $\left.\lambda_{n}>0\right)$ fitting into the $n \times n$ square. Let $I(\lambda) \in \mathbb{Z}$ be the one defined in Section 1 . We here recall the definition for the convenience of the reader. Let $s$ be the number of lower-right corners of $\lambda$, i.e., $s=\left|\left\{i \in[n] \mid \lambda_{i}>\lambda_{i+1}\right\}\right|$ where $\lambda_{n+1}:=0$. Write

$$
\left\{i \in[n] \mid \lambda_{i}>\lambda_{i+1}\right\}=\left\{i_{1}, \cdots, i_{s}\right\} .
$$

We impose the condition $i_{1}<i_{2}<\cdots<i_{s}$ to determine them uniquely. Observe that $i_{s}=n$. For $r=1, \cdots, s$, define

$$
a_{r}:=i_{r}-i_{r-1}-1, \quad b_{r}:=\lambda_{i_{r}}-\lambda_{i_{r+1}}-1, \quad c_{r}:=\lambda_{i_{r}}+i_{r}-n-1
$$

where we write $i_{0}=0$, and let

$$
y_{r}:=\left(\begin{array}{l}
a_{r} \\
c_{r}
\end{array}\right)\left(\begin{array}{l}
b_{r} \\
c_{r}
\end{array}\right) \quad \text { for } r=1, \cdots, s .
$$

See Figure 1 for the pictorial meaning of these numbers. We use the convention $\left(\begin{array}{l}x \\ y\end{array}\right)=0$ unless $0 \leqslant y \leqslant x$. Now, let

$$
I(\lambda):=(-1)^{n+s} y_{1} \cdots y_{s}
$$

The next is the main theorem of this section.

Theorem 7. If $S_{1}, \cdots, S_{n}$ form a nested chain of subsets, then we have

$$
\left(\mu_{X}, \tau_{S_{1}} \cdots \tau_{S_{n}}\right)=I(\lambda)
$$

where $\mu_{X}$ is the fundamental homology class and $\lambda$ is the Young diagram consisting of $\left|S_{1}\right|, \cdots,\left|S_{n}\right|$ reordered as a weakly decreasing sequence. Otherwise, the intersection number is zero. 
Proof. Recall that $\lambda$ is the Young diagram defined by $\lambda_{i}=\left|S_{n+1-i}\right|$ for $i=1, \cdots, n$. We denote $J(\lambda):=\left(\mu_{X}, \tau_{S_{1}} \cdots \tau_{S_{n}}\right)$, and we show that $J(\lambda)=I(\lambda)$. Observe that the condition $0 \leqslant c_{r} \leqslant b_{r}$ for all $r=1, \cdots, s$ is equivalent to the condition that each step of the zigzag line of the corners of $\lambda$ crosses the dotted anti-diagonal. If this condition is not satisfied, then both of $J(\lambda)$ and $I(\lambda)$ are zero. Hence, in the following, we can assume that this condition holds.

We prove the claim by induction on $k:=n-s$. For the case $k=0$, we have $\lambda_{i}=$ $\left|S_{n+1-i}\right|=n+1-i$ for all $1 \leqslant i \leqslant n$. So we have $J(\lambda)=1$ by $(4)$. Since $y_{1}=\cdots=y_{n}=1$ in this case, we have $I(\lambda)=1$, and the claim follows. For a general case, there is a lower-right corner (say $r$-th corner from the top) of $\lambda$ whose vertical line has length $\geqslant 2$. Then, Lemma 4 and Proposition 6 combined together show that

$$
J(\lambda)= \begin{cases}-\left(\begin{array}{c}
b_{r-1} \\
c_{r-1}
\end{array}\right) J\left(\lambda^{\prime}\right)-\left(\begin{array}{c}
b_{r} \\
c_{r}
\end{array}\right) J\left(\lambda^{\prime \prime}\right) & \left(\text { if } \lambda \neq \lambda^{\prime}, \lambda^{\prime \prime}\right) \\
-\left(\begin{array}{c}
b_{r-1} \\
c_{r-1}
\end{array}\right) J\left(\lambda^{\prime}\right) & \left(\text { if } \lambda \neq \lambda^{\prime}, \lambda=\lambda^{\prime \prime}\right) \\
-\left(\begin{array}{c}
b_{r} \\
c_{r}
\end{array}\right) J\left(\lambda^{\prime \prime}\right) & \left(\text { if } \lambda=\lambda^{\prime}, \lambda \neq \lambda^{\prime \prime}\right)\end{cases}
$$

where $a_{r}, b_{r}$, and $c_{r}$ are those for $\lambda$, and the Young diagrams $\lambda^{\prime}$ and $\lambda^{\prime \prime}$ are given by

$$
\lambda_{j}^{\prime}=\left\{\begin{array}{ll}
n+1-j & \text { if } j=i_{r-1}+1, \\
\lambda_{j} & \text { otherwise },
\end{array} \quad \lambda_{j}^{\prime \prime}= \begin{cases}n+1-j & \text { if } j=i_{r}, \\
\lambda_{j} & \text { otherwise }\end{cases}\right.
$$

(See Figure 3.) Note that there are no cases that $\lambda=\lambda^{\prime}=\lambda^{\prime \prime}$ since our vertical line has length $\geqslant 2$.
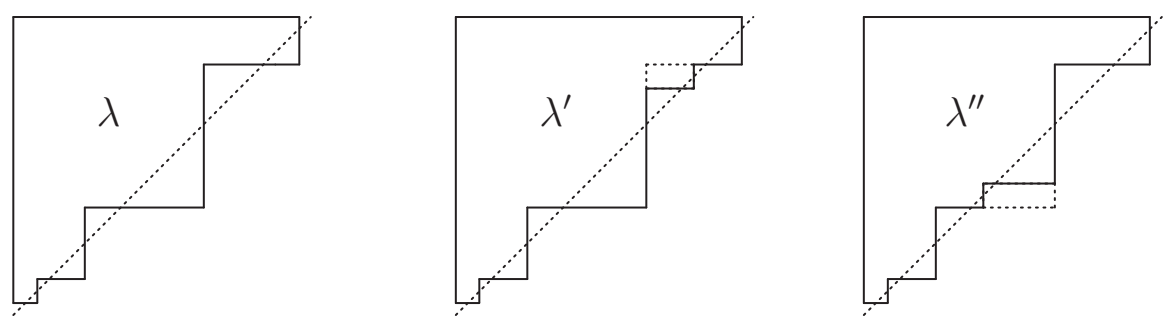

Figure 3: The Young diagrams $\lambda, \lambda^{\prime}$ and $\lambda^{\prime \prime}$

If $\lambda \neq \lambda^{\prime}$, by the induction hypothesis, we have

$$
J\left(\lambda^{\prime}\right)=(-1)^{n+(s+1)} y_{1} \cdots y_{r-2} \cdot\left(\begin{array}{c}
a_{r-1} \\
c_{r-1}
\end{array}\right) \cdot 1 \cdot\left(\begin{array}{c}
a_{r}-1 \\
c_{r}
\end{array}\right)\left(\begin{array}{c}
b_{r} \\
c_{r}
\end{array}\right) \cdot y_{r+1} \cdots y_{s} .
$$


Similarly, if $\lambda \neq \lambda^{\prime \prime}$, we have

$$
J\left(\lambda^{\prime \prime}\right)=(-1)^{n+(s+1)} y_{1} \cdots y_{r-1} \cdot\left(\begin{array}{c}
a_{r}-1 \\
c_{r}-1
\end{array}\right) \cdot 1 \cdot y_{r+1} \cdots y_{s} .
$$

Observe that the right-hand-sides of (11) and (12) vanish when $\lambda=\lambda^{\prime}$ and $\lambda=\lambda^{\prime \prime}$, respectively. Hence, the right-hand-side of the equation (10) can be written as

$$
\begin{aligned}
J(\lambda)= & -\left(\begin{array}{c}
b_{r-1} \\
c_{r-1}
\end{array}\right) \cdot(-1)^{n+s+1} y_{1} \cdots y_{r-2}\left(\begin{array}{c}
a_{r-1} \\
c_{r-1}
\end{array}\right)\left(\begin{array}{c}
a_{r}-1 \\
c_{r}
\end{array}\right)\left(\begin{array}{c}
b_{r} \\
c_{r}
\end{array}\right) y_{r+1} \cdots y_{s} \\
& -\left(\begin{array}{c}
b_{r} \\
c_{r}
\end{array}\right) \cdot(-1)^{n+s+1} y_{1} \cdots y_{r-2} y_{r-1}\left(\begin{array}{c}
a_{r}-1 \\
c_{r}-1
\end{array}\right) y_{r+1} \cdots y_{s} \\
= & (-1)^{n+s} y_{1} \cdots y_{s}=I(\lambda) .
\end{aligned}
$$

\section{The ring structure of the cohomology}

The cohomology ring $H^{*}(X)$ of the toric manifold $X$ associated with the fan $\Delta\left(A_{n}\right)$ is given by the face ring of $\Delta\left(A_{n}\right)$ modulo the linear relations (3) ([6]). As an application of Theorem 7, we describe the ring structure of the cohomology $H^{*}(X)$ in terms of an additive basis.

Recall that $D_{u \omega_{i}}$ for some $i \in[n]$ and permutation $u \in \mathfrak{S}_{n+1}$ is the invariant divisor of $X$ associated with the ray generated by $u \omega_{i} \in N$. Let

$$
X_{u}:=\bigcap_{i} D_{u \omega_{i}}
$$

for each permutation $u \in \mathfrak{S}_{n+1}$ where $i$ runs over all descents in $u$. Here, a descent in $u$ is a number $i \in[n]$ which satisfies $u(i)>u(i+1)$, and we denote by $d(u)$ the number of descents in $u$. Denote by $\left[X_{u}\right] \in H^{2 d(u)}(X)$ the Poincaré dual of $X_{u}$, then we have

$$
\left[X_{u}\right]=\prod_{i} \tau_{\{u(1), \cdots, u(i)\}}
$$

where $i$ runs over all descents in $u$ since invariant divisors of $X$ intersect transversely. $\left\{\left[X_{u}\right]\right\}_{u \in \mathfrak{S}_{n+1}}$ forms a module basis of $H^{*}(X)$ (See [7] or [1] for combinatorial proofs and [4] for a geometric proof). The class $\left[X_{u}\right]$ can be expressed by a Young diagram consisting of the descents in $u$ with the numbers in the nested chain of subsets in $D(u)$ (see (1) for the definition) written above the diagram so that each column represents the written number above it. This expression effectively encodes the descents in $u$ and the information of the chain of subsets. (See Figure 4.) Denoting $Y^{u}:=w_{0} X_{w_{0} u}=\cap X_{u[i]}$ where the intersection runs over all ascents $i$ in $u$, the similar expression works for $\left[Y^{u}\right]$ and the chain of subsets in $A(u)$. Here, $w_{0}$ is the longest element of $\mathfrak{S}_{n+1}$.

For $u, v, w \in \mathfrak{S}_{n+1}$, we have the Young diagram $\lambda_{u v}^{w}$ constructed in Section 1. Recall that $\mu_{X}$ is the fundamental homology class of $X$. The following corollary provides the combinatorial rule to compute the intersection number of $X_{u}, X_{v}$, and $Y^{w}$ in $X$. 


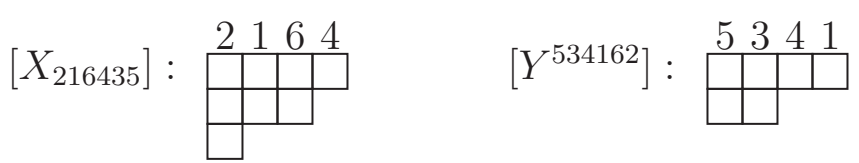

Figure 4: Two examples for $n=5$ in one-line notations

Corollary 8. $\left(\mu_{X},\left[Y^{w}\right]\left[X_{u}\right]\left[X_{v}\right]\right)=I\left(\lambda_{u v}^{w}\right)$.

For example, for $n=4$, we have

$$
\left(\mu_{X},\left[Y^{35421}\right]\left[X_{12354}\right]\left[X_{31254}\right]\right)=2 .
$$

In Figure 5, we left the numbers on the Young diagram so that we can see the nested chain of subsets appeared in the construction of $\lambda_{u v}^{w}$.

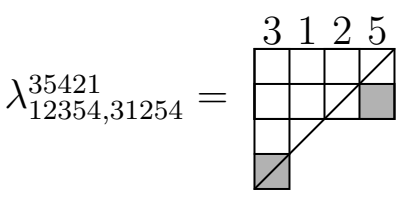

Figure 5:

Since $\left\{\left[X_{u}\right]\right\}_{u \in \mathfrak{S}_{n+1}}$ forms a module basis of $H^{*}(X)$, we can consider the expansion coefficients of the product

$$
\left[X_{u}\right]\left[X_{v}\right]=\sum_{w} c_{u v}^{w}\left[X_{w}\right]
$$

For example, these coefficients for the product $\left[X_{s_{i}}\right]\left[X_{s_{j}}\right]$ can be calculated directly if $|i-j| \geqslant 1$ where $s_{i}$ is the simple reflection exchanging $i$ and $i+1$. In fact, we have

$$
\left[X_{s_{i}}\right]\left[X_{s_{j}}\right]= \begin{cases}{\left[X_{s_{i} s_{j}}\right]\left(=\left[X_{s_{j} s_{i}}\right]\right)} & \text { if }|i-j| \geqslant 2, \\ 0 & \text { if }|i-j|=1 .\end{cases}
$$

since $\{1, \cdots, i-1, i+1\}$ and $\{1, \cdots, i, i+2\}$ do not form a chain of subsets.

Let us describe each structure constant $c_{u, v}^{w}$ in terms of intersection numbers computed above. Since a Weyl chamber $\sigma_{u}=\operatorname{cone}\left(u \omega_{1}, \cdots, u \omega_{n}\right)$ is a maximal cone of the fan, $\sigma_{u}$ is identified with a fixed point of the canonical torus action on $X$ denoted by $p_{u} \in X$ where $p_{u}$ is the intersection $\cap_{i=1}^{n} D_{u \omega_{i}}$. Then from the definition of $X_{w^{\prime}}$, one can show that $p_{u} \in X_{w^{\prime}}$ implies $u \geqslant w^{\prime}$ (e.g. [2]; Theorem 2.6.3) where $>$ is the Bruhat order. If $Y^{w} \cap X_{w^{\prime}} \neq \emptyset$, then $Y^{w} \cap X_{w^{\prime}}$ must contain a fixed point since it is an intersection of invariant divisors of $X$, and hence it follows that $w \geqslant w^{\prime}$. From this observation, we see that $Y^{w} \cap X_{w^{\prime}}=\emptyset$ unless $w \geqslant w^{\prime}$. Also, it is easy to see that $Y^{w}$ and $X_{w^{\prime}}$ 
intersect transversally when $w=w^{\prime}$. Recalling that the class $\left[Y^{w}\right]\left[X_{w^{\prime}}\right]$ is supported on the intersection $Y^{w} \cap X_{w^{\prime}}$, we obtain

$$
\left(\mu_{X},\left[Y^{w}\right]\left[X_{w^{\prime}}\right]\right)= \begin{cases}0 & \text { unless } w \geqslant w^{\prime} \text { and } d(w)=d\left(w^{\prime}\right) \\ 1 & \text { if } w=w^{\prime}\end{cases}
$$

See [4] for a proof using a cellular decomposition of $X$. Let $\mathcal{I}$ be the matrix whose $(u, v)$ component is given by $\mathcal{I}_{v}^{u}=\left(\mu_{X},\left[Y^{u}\right]\left[X_{v}\right]\right)=I\left(\lambda_{v i d}^{u}\right)$ for all $u, v \in W$. This matrix $\mathcal{I}$ is invertible over $\mathbb{Z}$ because of (15). Now, each coefficient $c_{u v}^{w}$ in (13) is a linear transform of the intersection numbers $I\left(\lambda_{u v}^{w}\right)$;

$$
c_{u v}^{w}=\sum_{w^{\prime}}\left(\mathcal{I}^{-1}\right)^{w}{ }_{w^{\prime}} I\left(\lambda_{u v}^{w^{\prime}}\right) .
$$

We note that it suffices to take the sum for $w^{\prime}$ satisfying $d(w)=d\left(w^{\prime}\right)$ and $w \geqslant w^{\prime}$ since $\left(\mathcal{I}^{-1}\right)_{w^{\prime}}^{w}$ is also upper-triangular in the sense of the right-hand-side of (15).

So the formula (16) exhibits the upper-triangularity of $c_{u v}^{w}$ in the sense that $c_{u v}^{w}=0$ unless $u, v \leqslant w$ since $I\left(\lambda_{u v}^{w}\right)$ satisfies the same property.

The transition formula (16) together with (15) provides us a recursive formula for the structure constants $c_{u v}^{w}$ which is manifestly integral;

$$
c_{u v}^{w}=I\left(\lambda_{u v}^{w}\right)-\sum_{w>w^{\prime}} \mathcal{I}_{w^{\prime}}^{w} c_{u v}^{w^{\prime}} .
$$

Note again that it is enough to take the sum for all $w^{\prime}$ satisfying $d(w)=d\left(w^{\prime}\right)$ and $w>w^{\prime}$. From this recursion, we recover (14), and we can compute the expansion of $\left[X_{s_{i}}\right]^{2}$. For example, if $n=3$, we obtain

$$
\left[X_{2134}\right]^{2}=\left[X_{2431}\right]-\left[X_{4213}\right]-\left[X_{3421}\right]-\left[X_{3241}\right]-\left[X_{3214}\right] .
$$

\section{$5 \quad$ Other classical types}

Note that the argument in the previous section can be naturally generalized to arbitrary root systems by considering the non-singular subvariety

$$
X_{u}=\bigcap_{i} D_{u \omega_{i}}
$$

for each $u \in W$ where $D_{u \omega_{i}}$ is the invariant divisor of $X$ corresponding to the ray generated by $u \omega_{i}$ and $i$ runs over all $i$ satisfying $u\left(\alpha_{i}\right) \in \Phi^{-}$. Here, $\Phi^{-}$is the set of negative roots. It follows that the Poincaré duals $\left\{\left[X_{u}\right]\right\}_{u \in W}$ form an additive basis of the integral cohomlogy $H^{*}(X)$ (see [4]).

Remark 9. The collections $\left\{c_{u, v}^{w}\right\}_{u, v, w \in W}$ and $\left\{\left(\mu_{X},\left[Y^{w}\right]\left[X_{u}\right]\left[X_{v}\right]\right)\right\}_{u, v, w \in W}$ are independent on the choice of the simple roots $\Pi$. 


\subsection{Intersection numbers for type $B_{n}$}

For the classical root system of type $B_{n}$, the roots are $\left\{t_{i}-t_{j}, \pm\left(t_{i}+t_{j}\right), \pm t_{i} \in E \mid 1 \leqslant\right.$ $i \neq j \leqslant n\}$ where $E=\mathbb{R}^{n}$. We choose $\Pi=\left\{t_{i}-t_{i+1}, t_{n} \mid 1 \leqslant i \leqslant n-1\right\}$ as a set of simple roots, and write $\alpha_{i}=t_{i}-t_{i+1}(1 \leqslant i \leqslant n-1), \alpha_{n}=t_{n}$. The Weyl group $\widetilde{\mathfrak{S}}_{n}$ is the $n$-th signed permutation group. Letting $t_{-i}:=-t_{i}$ for all $1 \leqslant i \leqslant n, u \in \widetilde{\mathfrak{S}}_{n}$ acts on $E$ by $u t_{i}=t_{u(i)}$. The minimal generators $\omega_{1}, \cdots, \omega_{n} \in E^{*}$ of the fundamental Weyl chamber are $\omega_{i}=e_{1}+\cdots+e_{i}$ for $i=1, \cdots, n$.

Let $[ \pm n]=\{1, \cdots, n,-1, \cdots,-n\}$. For $S \in 2^{[ \pm n]}$, consider a condition

$$
\text { for any } i \in[ \pm n] \text {, if } i \in S \text { then }-i \notin S \text {. }
$$

We have a well-defined map $\Phi^{*} \rightarrow 2^{[ \pm n]}$ by $u \omega_{i} \mapsto\{u(1), \cdots, u(i)\}$. This leads us to an identification

$$
\Phi^{*} \longleftrightarrow \quad \text { the set of non-empty subsets of }[ \pm n] \text { satisfying }(*) \text {. }
$$

Now, for each $\emptyset \subsetneq S \subset[ \pm n]$ satisfying $(*)$, we define $\tau_{S}:=\tau_{u \omega_{i}}$ where $u \omega_{i} \in \Phi^{*}$ corresponds to $S$ by this identification. For $\emptyset \subsetneq S_{1}, \cdots, S_{q} \subset[ \pm n](1 \leqslant q \leqslant n)$ satisfying $(*)$, we have that $\tau_{S_{1}} \cdots \tau_{S_{q}}=0$ unless these sets form a nested chain of subsets, as in the case for type $A_{n}$.

For each $k \in[ \pm n]$, let $B \subset[ \pm n]$ satisfy $(*), k \in B$, and $|B|=n$. From the linear relation (3) for the root $\alpha=t_{k}$, we can deduce that

$$
\tau_{B}^{2}=-\sum_{\substack{k \in B^{\prime} \\ B^{\prime} \subsetneq B}} \tau_{B^{\prime}} \tau_{B}
$$

where the sum is taken over all $\emptyset \subsetneq B^{\prime} \subset[ \pm n]$ satisfying $(*)$ with the prescribed conditions. Similarly, for each $k, l \in[ \pm n]$, let $B \subset[ \pm n]$ satisfy $(*), k \in B$, and $\pm l \notin B$ (hence $1 \leqslant|B| \leqslant n-1$ ). Then from (3) for the root $\alpha=t_{k}-t_{l}$, we obtain

$$
\tau_{B}^{2}=-\sum_{\substack{k \in B^{\prime}, \pm l \notin B^{\prime} \\ B^{\prime} \neq B}} \tau_{B^{\prime}} \tau_{B}-\sum_{k,-l \in B^{\prime}} 2 \tau_{B^{\prime}} \tau_{B} .
$$

Observe that the second summand will vanish after multiplying $\tau_{A}$ and $\tau_{C}$ for $A \subset B \backslash\{k\}$ and $B \coprod\{l\} \subset C$ where we write $\tau_{\emptyset}=0$. So these two equations can be used to prove the separation rule similar to Lemma 5 , and we obtain the same type of vanishing property as in Proposition 6. Now the argument in the proof of Theorem 7 also works for this case, and it follows that

Theorem 10. If $\emptyset \subsetneq S_{1}, \cdots, S_{n} \subset[ \pm n]$ satisfying $(*)$ form a nested chain of subsets, then we have

$$
\left(\mu_{X}, \tau_{S_{1}} \cdots \tau_{S_{n}}\right)=2^{n-\lambda_{1}} I(\lambda)
$$

where $\mu_{X}$ is the fundamental homology class of $X$ and $\lambda$ is the Young diagram consisting of $\left|S_{1}\right|, \cdots,\left|S_{n}\right|$ reordered as a weakly decreasing sequence and $I$ is the function defined in (9). Otherwise, the intersection number is zero. 
Let $\alpha_{i}:=t_{i}-t_{i+1}$ for $1 \leqslant i \leqslant n-1$ and $\alpha_{n}:=t_{n}$. For each signed permutation $u \in \widetilde{\mathfrak{S}}_{n}$, an element $i \in[n]$ satisfies $u\left(\alpha_{i}\right) \in \Phi^{-}$if and only if

(D-1) if $i \leqslant n-1$, then $u(i)>u(i+1)$ with the same sign or $u(i)<u(i+1)$ with different signs,

(D-2) if $i=n$, then $u(i)<0$.

Similarly, consider the conditions

(A-1) if $i \leqslant n-1$, then $u(i)<u(i+1)$ with the same sign or $u(i)>u(i+1)$ with different signs,

$(\mathrm{A}-2)$ if $i=n$, then $u(i)>0$.

Denoting

$$
D(u):=\{u[i] \mid i \text { satisfies }(\mathrm{D})\} \quad \text { and } \quad A(u):=\{u[i] \mid i \text { satisfies }(\mathrm{A})\},
$$

we define a Young diagram $\lambda_{u, v}^{w}$ in the manner described in the last section. Note that we put $I(\emptyset)=0$ as a convention.

Now, for signed permutations $u, v, w \in \widetilde{\mathfrak{S}}_{n}$, the intersection number of $Y^{w}, X_{u}$, and $X_{v}$ in $X$ of type $B_{n}$ is given by the following.

Corollary 11. For signed permutations $u, v, w \in \widetilde{\mathfrak{S}}_{n}$, we have

$$
\left(\mu_{X},\left[Y^{w}\right]\left[X_{u}\right]\left[X_{v}\right]\right)=2^{n-\left(\lambda_{u, v}^{w}\right)_{1}} I\left(\lambda_{u v}^{w}\right)
$$

where I is the function defined in (9).

For example, for $n=4$ with the convention $\bar{k}=-k$, Corollary 11 computes

$$
\left(\mu_{X},\left[Y^{2 \overline{3} \overline{1} \overline{4}}\right]\left[X_{2 \overline{3} 14}\right]\left[X_{2 \overline{3} 14}\right]\right)=4 .
$$

(See Figure 6.)

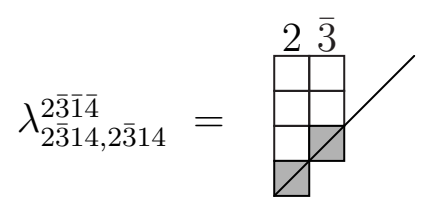

Figure 6: An example for type $B_{n}$ 


\subsection{Intersection numbers for type $C_{n}$}

For the classical root system of type $C_{n}$, the roots are $\left\{t_{i}-t_{j}, \pm\left(t_{i}+t_{j}\right), \pm 2 t_{i} \in E\right.$ | $1 \leqslant i \neq j \leqslant n\}$ where $E=\mathbb{R}^{n}$. We choose $\Pi=\left\{t_{i}-t_{i+1}, 2 t_{n} \mid 1 \leqslant i \leqslant n-1\right\}$ as a set of simple roots, and write $\alpha_{i}=t_{i}-t_{i+1}(1 \leqslant i \leqslant n-1), \alpha_{n}=2 t_{n}$. The Weyl group $\widetilde{\mathfrak{S}}_{n}$ is the $n$-th signed permutation group as above. The minimal generators $\omega_{1}, \cdots, \omega_{n}$ of the fundamental Weyl chamber are $\omega_{i}=e_{1}+\cdots+e_{i}$ for $i=1, \cdots, n-1$ and $\omega_{n}=\frac{1}{2}\left(e_{1}+\cdots+e_{n}\right)$.

We have a well-defined map $\Phi^{*} \rightarrow 2^{[ \pm n]}$ by $v \omega_{i} \mapsto\{v(1), \cdots, v(i)\}$, and obtain an identification $\Phi^{*}$ and the set of non-empty subsets of $[ \pm n]$ satisfying $(*)$. For $\emptyset \subsetneq S_{1}, \cdots, S_{q} \subsetneq$ $[ \pm n](1 \leqslant q \leqslant n)$ satisfying $(*)$, we have that $\tau_{S_{1}} \cdots \tau_{S_{q}}=0$ unless these sets form a nested chain of subsets where $\tau_{S}$ is defined as in Section 5.1.

For each $k \in[ \pm n]$, let $B \subset[ \pm n]$ satisfy $(*), k \in B$, and $|B|=n$. Then (3) for the root $\alpha=2 t_{k}$ shows that

$$
\tau_{B}^{2}=-\sum_{\substack{k \in B^{\prime} \\ B^{\prime} \subsetneq B}} 2 \tau_{B^{\prime}} \tau_{B}
$$

where the sum is taken over all $\emptyset \subsetneq B^{\prime} \subset[ \pm n]$ satisfying $(*)$ with the prescribed conditions. For each $k, l \in[ \pm n]$, let $B \subset[ \pm n]$ satisfy $(*), k \in B$, and $\pm l \notin B$ (hence $1 \leqslant|B| \leqslant n-1$ ). Then from (3) for the root $\alpha=t_{k}-t_{l}$, we obtain

$$
\tau_{B}{ }^{2}=-\sum_{\substack{k \in B^{\prime}, \pm l \notin B^{\prime} \\ B^{\prime} \neq B}} \tau_{B^{\prime}} \tau_{B}-\sum_{\substack{k \in B^{\prime},-l \in B^{\prime} \\\left|B^{\prime}\right| \neq n}} 2 \tau_{B^{\prime}} \tau_{B}-\sum_{\substack{k \in B^{\prime},-l \in B^{\prime} \\\left|B^{\prime}\right|=n}} \tau_{B^{\prime}} \tau_{B} .
$$

With a similar observation made for type $B_{n}$, we again have the same type of vanishing property as in Proposition 6. Hence, we obtain

Theorem 12. If $\emptyset \subsetneq S_{1}, \cdots, S_{n} \subsetneq[ \pm n]$ satisfying $(*)$ form a nested chain of subsets, then we have

$$
\left(\mu_{X}, \tau_{S_{1}} \cdots \tau_{S_{n}}\right)=2^{n-\lambda_{1}+m-1} I(\lambda)
$$

where $\mu_{X}$ is the fundamental homology class of $X$ and $\lambda$ is the Young diagram consisting of the numbers $\left|S_{1}\right|, \cdots,\left|S_{n}\right|$ reordered as a weakly decreasing sequence and $I$ is the function defined in (9) and $m$ is the number of rows of $\lambda$ of length $n$. Otherwise, the intersection number is zero.

For signed permutations $u, v, w \in \widetilde{\mathfrak{S}}_{n}$, let $\lambda_{u, v}^{w}$ be the Young diagram defined in Section 5.1. The intersection number of $Y^{w}, X_{u}$, and $X_{v}$ in $X$ of type $C_{n}$ is given by the following.

Corollary 13. For signed permutations $u, v, w \in \widetilde{\mathfrak{S}}_{n}$, we have

$$
\left(\mu_{X},\left[Y^{w}\right]\left[X_{u}\right]\left[X_{v}\right]\right)=2^{n-\left(\lambda_{u, v}^{w}\right)_{1}+m-1} I\left(\lambda_{u v}^{w}\right)
$$

where $I$ is the function defined in (9) and and $m$ is the number of rows of $\lambda_{u, v}^{w}$ of length $n$. 


\subsection{Intersection numbers for type $D_{n}$}

For the classical root system of type $D_{n}$, the roots are $\left\{t_{i}-t_{j}, \pm\left(t_{i}+t_{j}\right) \in E \mid 1 \leqslant i \neq\right.$ $j \leqslant n\}$ where $E=\mathbb{R}^{n}$. We choose $\Pi=\left\{t_{i}-t_{i+1}, t_{n-1}+t_{n} \mid 1 \leqslant i \leqslant n-1\right\}$ as a set of simple roots, and write $\alpha_{i}=t_{i}-t_{i+1}(1 \leqslant i \leqslant n-2), \alpha_{n-1}=t_{n-1}+t_{n}, \alpha_{n}=t_{n-1}-t_{n}$. The Weyl group $\widetilde{\mathfrak{S}}_{n}^{+}$is the $n$-th even signed permutation group defined by

$$
\widetilde{\mathfrak{S}}_{n}^{+}:=\left\{w \in \widetilde{\mathfrak{S}}_{n} \mid \text { the number of } i \text { with } w(i)<0 \text { is even }\right\}
$$

where $\widetilde{\mathfrak{S}}_{n}$ is the $n$-th signed permutation group. The minimal generators $\omega_{1}, \cdots, \omega_{n}$ $\in E^{*}$ of the fundamental Weyl chamber are $\omega_{i}=e_{1}+\cdots+e_{i}$ for $i=1, \cdots, n-2$, $\omega_{n-1}=\frac{1}{2}\left(e_{1}+\cdots+e_{n-1}+e_{n}\right)$ and $\omega_{n}=\frac{1}{2}\left(e_{1}+\cdots+e_{n-1}-e_{n}\right)$.

For $S \in 2^{[ \pm n]}$, consider a condition

$$
|S| \neq n-1 \text {, and if } i \in S \text { then }-i \notin S \text { for any } i \in[ \pm n] .
$$

We have a well-defined map $\Phi^{*} \rightarrow 2^{[ \pm n]}$ given by

$$
\begin{aligned}
& u \omega_{i} \mapsto u[i]=\{u(1), \cdots, u(i)\} \quad \text { for } 1 \leqslant i \leqslant n-2, \\
& u \omega_{n-1} \mapsto u[n]_{+}=\{u(1), \cdots, u(n-1), u(n)\}, \\
& u \omega_{n} \mapsto u[n]_{-}=\{u(1), \cdots, u(n-1),-u(n)\} .
\end{aligned}
$$

where $[n]_{+}=\{1,2, \cdots, n-1, n\}$ and $[n]_{-}=\{1,2, \cdots, n-1,-n\}$.

It follows that this map $\Phi^{*} \rightarrow 2^{[ \pm n]}$ is an injection. In fact, we cannot have

$$
\{u(1), \cdots, u(n-1), u(n)\}=\{v(1), \cdots, v(n-1),-v(n)\}
$$

for any $u, v \in \widetilde{\mathfrak{S}}_{n}^{+}$since the number of negative integers in the left hand side and the righthand-side are different, and so $u \omega_{n-1}$ and $v \omega_{n}$ are never mapped to the same element. The other cases are left to the reader. So we can make an identification

$$
\Phi^{*} \longleftrightarrow \quad \text { the set of non-empty subsets of }[ \pm n] \text { satisfying }(* *)
$$

Hence, for each $\emptyset \subsetneq S \subset[ \pm n]$ satisfying $(* *)$, we define $\tau_{S}:=\tau_{u \omega_{i}}$ where $u \omega_{i} \in \Phi^{*}$ corresponds to $S$ by this identification.

Let us denote by $\mathcal{C}$ the set of chains of subsets $\left\{S_{i}^{\circ}\right\}_{i}$ of $[ \pm n]$ of the following form: there exists $u \in \widetilde{\mathfrak{S}}_{n}^{+}$such that $S_{i}^{\circ}=u[i]$ for $1 \leqslant i \leqslant n-2, S_{n-1}^{\circ}=u[n]_{+}$, and $S_{n}^{\circ}=u[n]_{-}$. Note that $\left\{S_{i}^{\circ}\right\}_{i}$ does not have a set of order $n-1$ and satisfies the same inclusion relation shown in Figure 7. A subchain $\left\{S_{i}\right\}_{i}$ of a chain $\left\{S_{i}^{\circ}\right\}_{i}$ in $\mathcal{C}$ is a sequence satisfying $S_{j} \in\left\{S_{i}^{\circ}\right\}_{i}$ for $1 \leqslant j \leqslant n$ and $S_{j} \subset S_{j^{\prime}}$ for $1 \leqslant j \leqslant j^{\prime} \leqslant n$ unless $\left|S_{j}\right|=\left|S_{j^{\prime}}\right|=n$. For $\emptyset \subsetneq S_{1}, \cdots, S_{q} \subsetneq[ \pm n](1 \leqslant q \leqslant n)$ satisfying $(* *)$, we have $\tau_{S_{1}} \cdots \tau_{S_{q}}=0$ unless the sequence forms a subchain of a chain in $\mathcal{C}$ up to reordering. Let $\left\{S_{i}\right\}_{i}$ be a subchain of a chain in $\mathcal{C}$. For $S_{i}$ satisfying $\left|S_{i}\right|=n$, we say that $S_{i}$ is even (resp. odd) if the number of negative elements of $S_{i}$ is even (resp. odd). Recall that $\mu_{X}$ is the fundamental homology class of $X$. The following is Lemma 3 for type $D_{n}$. 


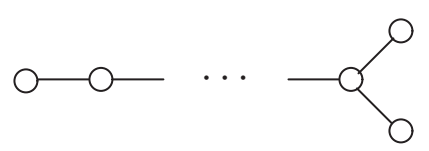

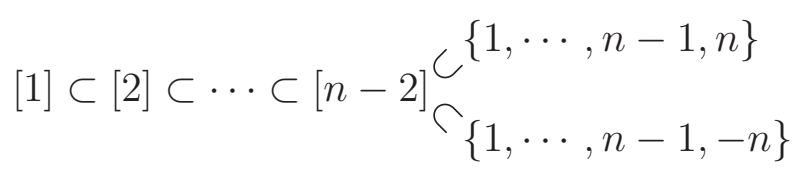

Figure 7: The Dynkin diagram and a maximal chain of subsets for type $D_{n}$

Lemma 14. Let $\left\{S_{i}\right\}_{i}$ and $\left\{S_{i}^{\prime}\right\}_{i}$ be subchains of some chains in $\mathcal{C}$. If $\left|S_{i}\right|=\left|S_{i}^{\prime}\right|$ for $i=1, \cdots, n$ and the number of even $S_{i}$ 's and the number of even $S_{i}^{\prime}$ 's are the same, then $\left(\mu_{X}, \tau_{S_{1}} \cdots \tau_{S_{n}}\right)=\left(\mu_{X}, \tau_{S_{1}^{\prime}} \cdots \tau_{S_{n}^{\prime}}\right)$.

Let $\left\{S_{i}\right\}_{i}$ be a subchain of a chain in $\mathcal{C}$. We denote by $\lambda$ the signed Young diagram consisting of $\lambda_{i}=\left|S_{n+1-i}\right|$ for $i=1, \cdots, n$ where the label of $\lambda$ is defined as follows: if we have $\lambda_{i}=n$, then we label this row by + (resp. -) if $S_{n+1-i}$ is even (resp. odd). Recall from Section 3 that the dotted anti-diagonal line drawn on the Young diagram is the dotted line shifted down half the length of a single box from the standard anti-diagonal. Our first aim is to prove the following.

Proposition 15. (The vanishing property) $\left(\mu_{X}, \tau_{S_{1}} \cdots \tau_{S_{n}}\right)=0$ unless each step of the zigzag line of the lower-right corners of $\lambda$ crosses the dotted anti-diagonal.
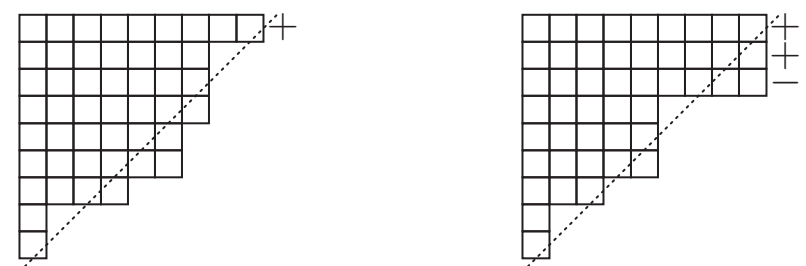

For each $k, l \in[ \pm n]$, let $B \subset[ \pm n]$ satisfy $(* *), k \in B$, and $\pm l \notin B$ (hence $|B| \leqslant n-2$ ). By the linear relation (3) for the root $\alpha=t_{k}-t_{l}$, it follows that

$$
\tau_{B}^{2}=-\sum_{\substack{k \in B^{\prime}, \pm l \notin B^{\prime} \\ B^{\prime} \neq B}} \tau_{B^{\prime}} \tau_{B}-\sum_{\substack{k \in B^{\prime},-l \in B^{\prime} \\\left|B^{\prime}\right| \neq n}} 2 \tau_{B^{\prime}} \tau_{B}-\sum_{\substack{k \in B^{\prime},-l \in B^{\prime} \\\left|B^{\prime}\right|=n}} \tau_{B^{\prime}} \tau_{B}
$$

where the sum is taken over all $\emptyset \subsetneq B^{\prime} \subset[ \pm n]$ satisfying $(* *)$ with the prescribed conditions. If $A \subsetneq B$ with $k \notin A$, and if $B \subsetneq C$ with $l \in C$ then,

$$
\tau_{A} \tau_{B}{ }^{2} \tau_{C}=-\sum_{\substack{k \in B^{\prime}, \pm l \notin B^{\prime} \\ A \subseteq B^{\prime} \subsetneq C, B^{\prime} \neq B}} \tau_{A} \tau_{B^{\prime}} \tau_{B} \tau_{C}-\delta_{|C|, n} \tau_{A} \tau_{B} \tau_{(l,-l) C} \tau_{C} .
$$

where $\delta_{|C|, n}$ is the Kronecker delta. If $|C|=n$, then after multiplying (20) by $\tau_{\bar{C}}$ where $\bar{C}=(p,-p) C$ for some $p \in C \backslash\{l\}$, we obtain

$$
\tau_{A} \tau_{B}^{2} \tau_{C} \tau_{\bar{C}}=-\sum_{\substack{k \in B^{\prime}, \pm l, \pm p \notin B^{\prime} \\ A \subsetneq B^{\prime} \subsetneq C, B^{\prime} \neq B}} \tau_{A} \tau_{B^{\prime}} \tau_{B} \tau_{C} \tau_{\bar{C}} .
$$


Let $\lambda$ as above. We denote

$$
\begin{aligned}
& m_{+}(\lambda):=\mid\left\{i \mid \lambda_{i}=n \text { and the label of } \lambda_{i} \text { is }+\right\} \mid, \\
& m_{-}(\lambda):=\mid\left\{i \mid \lambda_{i}=n \text { and the label of } \lambda_{i} \text { is }-\right\} \mid .
\end{aligned}
$$

Lemma 16. Suppose that one of the following holds:

(i) $m_{+}(\lambda)=m_{-}(\lambda)=1$

(ii) $\left(m_{+}(\lambda), m_{-}(\lambda)\right)$ is equal to $(1,0)$ or $(0,1)$,

(iii) $m_{+}(\lambda)=m_{-}(\lambda)=0$.

Then $\left(\mu_{X}, \tau_{S_{1}} \cdots \tau_{S_{n}}\right)=0$ unless each step of the zigzag line of the corners of $\lambda$ crosses the dotted anti-diagonal.

Proof. The claim for the case (i) can be proved by (20) and (21) as in the proof of Proposition 6. For the case (ii), the same argument works together with (20), since we have already proved the claim for the case (i). Now, the case (iii) is shown again by the same proof used for Proposition 6 together with (19), (20), and the case (ii).

For each $k, l \in[ \pm n]$, let $\emptyset \subsetneq B \subsetneq[ \pm n]$ satisfy $(* *), k, l \in B$, and $|B|=n$. If $A \subset B \backslash\{k, l\}$, then from the linear relation (3) for the root $\alpha=t_{k}+t_{l}$, we obtain

$$
\begin{aligned}
& \tau_{A} \tau_{B}^{2}=- \sum_{k \in B^{\prime}, \pm l \notin B^{\prime}} \tau_{A} \tau_{B^{\prime}} \tau_{B}-\sum_{\substack{ \pm \notin B^{\prime}, l \in B^{\prime}\\
}} \tau_{A} \tau_{B^{\prime}} \tau_{B} \\
&-\sum_{\substack{k, l \in B^{\prime},\left|B^{\prime}\right| \neq n}} 2 \tau_{A} \tau_{B^{\prime}} \tau_{B}-\sum_{\substack{k, l \in B^{\prime}, B^{\prime} \neq B,\left|B^{\prime}\right|=n}} \tau_{A} \tau_{B^{\prime}} \tau_{B}
\end{aligned}
$$

where we denote $\tau_{\emptyset}=1$. Especially if $A=B \backslash\{k, l\}$, then $|A|=n-2$ and we have

$$
\tau_{A} \tau_{B}^{2}=-\sum_{\substack{k, l \in B^{\prime}, B^{\prime} \neq B,\left|B^{\prime}\right|=n}} \tau_{A} \tau_{B^{\prime}} \tau_{B}=0 .
$$

The second equality follows since an element of $B^{\prime}$ which is neither $k$ nor $l$ has to be -1 times an element of $B$, which implies that $A \not \subset B^{\prime}$. On the other hand, letting $\bar{B}=(-k, k) B$, we obtain from (22) that

$$
\tau_{A} \tau_{B}^{2} \tau_{\bar{B}}=-\sum_{\substack{ \pm k \notin B^{\prime}, l \in B^{\prime} \\ A \subsetneq B^{\prime} \subsetneq B}} \tau_{A} \tau_{B^{\prime}} \tau_{B} \tau_{\bar{B}} .
$$

Lemma 17. Suppose that one of the following holds:

(i) $m_{+}(\lambda), m_{-}(\lambda) \geqslant 1$,

(ii) $m_{+}(\lambda) \geqslant 1$ and $m_{-}(\lambda)=0$, 
(iii) $m_{+}(\lambda)=0$ and $m_{-}(\lambda) \geqslant 1$.

Then $\left(\mu_{X}, \tau_{S_{1}} \cdots \tau_{S_{n}}\right)=0$ unless each step of the zigzag line of the lower-right corners of $\lambda$ crosses the dotted anti-diagonal.

Proof. The claim for the case (i) follows from (24) and the case (i) of Lemma 16 by induction on $m_{+}(\lambda)+m_{-}(\lambda)$. Let us consider the case (ii). We prove the claim by induction on $m_{+}(\lambda)$. For the case $m_{+}(\lambda)=1$, the claim follows from the case (ii) of Lemma 16. For the general case, the induction hypothesis and the claim for the case (i) shows our claim by applying (22) to reduce the multiplicity for $\tau_{S_{n}}$ in $\tau_{S_{1}} \cdots \tau_{S_{n}}$. The claim for the case (iii) can be proved similarly.

Now, Proposition 15 follows from Lemma 17 and the case (iii) of Lemma 16.

For a signed Young diagram $\lambda$ with $n$ rows fitting into the $n \times n$-square, let

$$
m:=\left|\left\{i \mid \lambda_{i}=n\right\}\right|=m_{+}(\lambda)+m_{-}(\lambda)
$$

be the number of rows of $\lambda$ of length $n$. Recall that the numbers $a_{r}, b_{r}, c_{r}$, and $y_{r}$ are defined in (7) and (8). We now define

$$
\widetilde{y}_{1}:= \begin{cases}2^{\left(n-\lambda_{1}-1\right)(1-m)}\left(\begin{array}{l}
a_{1} \\
c_{1}
\end{array}\right)\left(\begin{array}{l}
b_{1} \\
c_{1}
\end{array}\right) & \text { if } m \leqslant 1, \\
-\left(\begin{array}{c}
b_{1}-1 \\
c_{1}-1
\end{array}\right) & \text { if } m \geqslant 2 \text { and } m_{+}(\lambda) m_{-}(\lambda) \neq 0, \\
\left(2^{a_{1}}-a_{1}-1\right)\left(\begin{array}{l}
b_{1} \\
c_{1}
\end{array}\right)+\left(\begin{array}{c}
b_{1}-1 \\
c_{1}
\end{array}\right) & \text { if } m \geqslant 2 \text { and } m_{+}(\lambda) m_{-}(\lambda)=0\end{cases}
$$

Theorem 18. If $\emptyset \subsetneq S_{1}, \cdots, S_{n} \subsetneq[ \pm n]$ satisfying $(* *)$ form a subchain of a chain in $\mathcal{C}$, then we have

$$
\left(\mu_{X}, \tau_{S_{1}} \cdots \tau_{S_{n}}\right)=(-1)^{n+s} \widetilde{y}_{1} y_{2} \cdots y_{s}
$$

where $\mu_{X}$ is the fundamental homology class of $X$ and $\lambda$ is the signed Young diagram consisting of $\left|S_{1}\right|, \cdots,\left|S_{n}\right|$ reordered as a weakly decreasing sequence and $m$ is the number of rows of $\lambda$ of length $n$. Otherwise, the intersection number is zero.

Remark 19. In each case, the given number vanishes unless each step of the zigzag line of the lower-right corners of $\lambda$ crosses the dotted anti-diagonal.

Proof of Theorem 18. We compute the intersection number

$$
J(\lambda):=(-1)^{n-s}\left(\mu_{X}, \tau_{S_{1}} \cdots \tau_{S_{n}}\right)
$$

with sign where we can assume that each step of the zigzag line of the corners of $\lambda$ crosses the dotted anti-diagonal by Proposition 15 and the remark above. We first prove the 
case (i). If $m_{+}(\lambda)=1$ and $m_{-}(\lambda)=0$ (or $m_{+}(\lambda)=1$ and $m_{-}(\lambda)=0$ ), then it follows that $J(\lambda)=y_{1} \cdots y_{s}$. This can be proved by induction similar to that used in the proof of Theorem 7 because of the separating properties (20). So, let us consider the case of $m_{+}(\lambda)=m_{-}(\lambda)=0$. In this case, Proposition 15 shows that the separation rule (19) replaces the square $\tau_{S_{n}}^{2}$ in $\tau_{S_{1}} \cdots \tau_{S_{n}}$ to

$$
\sum_{\substack{k \in S^{\prime}, \pm l \notin S^{\prime} \\ S^{\prime} \subsetneq S_{n},\left|B^{\prime}\right| \neq n}} \tau_{S^{\prime}} \tau_{S_{n}}+\sum_{\substack{k \in S^{\prime},-l \in S^{\prime} \\\left|S^{\prime}\right|=n}} \tau_{S^{\prime}} \tau_{S_{n}}
$$

for some $k \in S_{n}$ and $\pm l \in S_{n}$ when we compute the intersection number $J(\lambda)$ with sign. Namely, this replacement can be pictured as

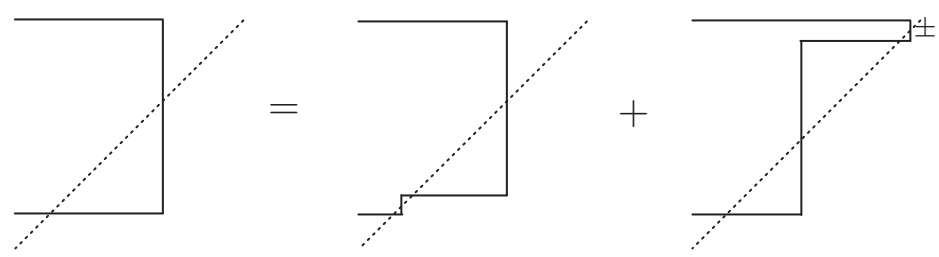

where we omit the coefficients in the picture. Hence, with the claim for the previous case, we get $J(\lambda)=2^{n-\lambda_{1}-1} y_{1} \cdots y_{s}$ as in the case of type $B_{n}$.

Let us consider the case (ii-a). We prove the claim by induction on the sum of the multiplicities for $S_{i}$ 's satisfying $\left|S_{i}\right| \neq n$. The base case has $\lambda_{i}=n+1-i$ for all $\lambda_{i} \neq n$, so it is obvious that $-J(\lambda)=(-1)^{n-s-1}\left(\mu_{X}, \tau_{S_{1}} \cdots \tau_{S_{n}}\right)$ is equal to 1 by iterating $(24)$. For the general case, we apply (20) and (21) to some square $\tau_{S_{i}}{ }^{2}$ with $\left|S_{i}\right| \neq n$ in $J(\lambda)$. If $i_{2}<i$, the computation with (20) works as in the proof of Theorem 7 . If $i_{1}<i \leqslant i_{2}$, then the right-hand-side of $(21)$ applied to $-J(\lambda)$ can be calculated as follows by the induction hypothesis:

$$
\left(\begin{array}{l}
b_{2} \\
c_{2}
\end{array}\right) \cdot\left(\begin{array}{c}
b_{1}-1 \\
c_{1}-1
\end{array}\right)\left(\begin{array}{c}
a_{2}-1 \\
c_{2}-1
\end{array}\right) y_{3} \cdots y_{s}+\left(\begin{array}{c}
b_{1}-1 \\
c_{1}-1
\end{array}\right) \cdot\left(\begin{array}{c}
a_{2}-1 \\
c_{2}
\end{array}\right)\left(\begin{array}{c}
b_{2} \\
c_{2}
\end{array}\right) y_{3} \cdots y_{s}
$$

which is equal to $\left(\begin{array}{l}b_{1}-1 \\ c_{1}-1\end{array}\right) y_{2} \cdots y_{s}$, and the claim follows.

Let us consider the case (ii-b). We can assume $m=m_{+}(\lambda)\left(=a_{1}+1\right)$ without loss of generality. We first consider the case that $\lambda_{i}=n+1-i$ for all $\lambda_{i} \neq n$. Note that $\left(\begin{array}{c}b_{1}-1 \\ c_{1}\end{array}\right)=0$ in this case. For this special case, we prove the claim by induction on $m=m_{+}(\lambda)$. For the case $m=a_{1}+1=2$, the intersection number is zero by (23), and the claim follows since $2^{a_{1}}-a_{1}-1=0$. For the general case, we have an inductive formula by (22), namely,
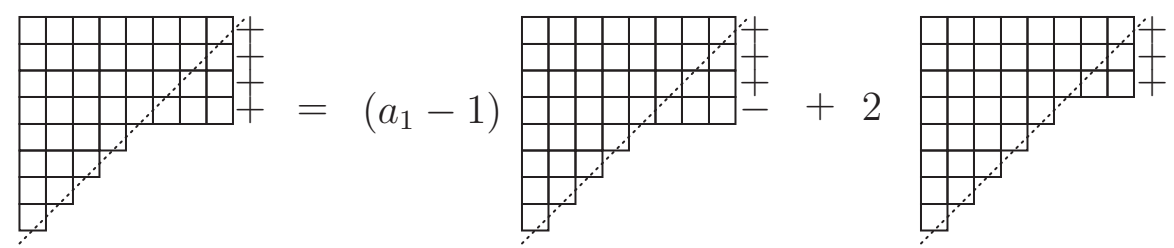
where the first coefficient $a_{1}-1$ comes from the choices of an element in $B^{\prime} \backslash(A \cup\{k, l\})$ turned to be negative, and the second coefficient 2 comes from the two summands corresponding to $k \in B^{\prime}, \pm l \notin B^{\prime}$ and $\pm k \notin B^{\prime}, l \in B^{\prime}$. Noticing that the intersection number for the first summand is equal to 1 as we already saw, it follows that

$$
J(\lambda)=\sum_{i=0}^{a_{1}} 2^{i}\left(a_{1}-1-i\right)=2^{a_{1}}-a_{1}-1 .
$$

We now prove the claim (ii-b) by induction on the sum of the multiplicities for $S_{i}$ satisfying $\left|S_{i}\right| \neq n$. The base case $\lambda_{i}=n+1-i$ for all $\lambda_{i} \neq n$ is proved above. For the general case, we apply (20). If $i_{2}<i$, the computation with (20) again works as in the proof of Theorem 7 . If $i_{1}<i \leqslant i_{2}$, we also apply (20) to a square $S_{i}^{2}$ in $-J(\lambda)$. Namely, we have
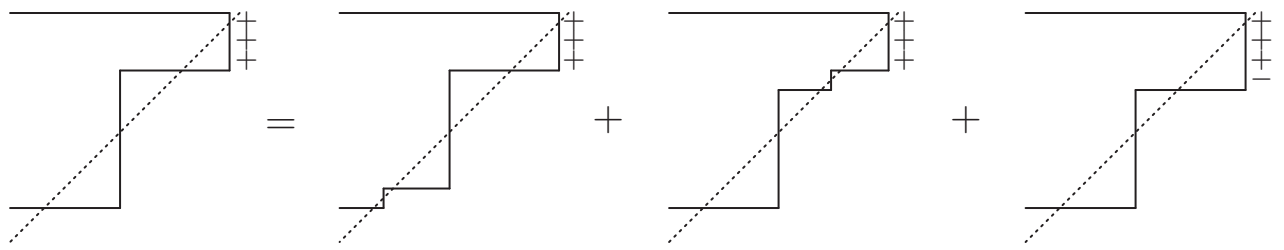

with omitting the coefficients. The right-hand-side can be calculated by the induction hypothesis and the claim for the case (ii-a), and $J(\lambda)=(-1)^{n-s}\left(\mu_{X}, \tau_{S_{1}} \cdots \tau_{S_{n}}\right)$ is

$$
\begin{aligned}
&\left(\begin{array}{c}
b_{2} \\
c_{2}
\end{array}\right) \cdot\left\{\left(2^{a_{1}}-a_{1}-1\right)\left(\begin{array}{l}
b_{1} \\
c_{1}
\end{array}\right)\left(\begin{array}{c}
a_{2}-1 \\
c_{2}-1
\end{array}\right) y_{3} \cdots y_{s}+\left(\begin{array}{c}
b_{1}-1 \\
c_{1}
\end{array}\right)\left(\begin{array}{c}
a_{2}-1 \\
c_{2}-1
\end{array}\right) y_{3} \cdots y_{s}\right\} \\
&+\left(\begin{array}{c}
b_{1} \\
c_{1}
\end{array}\right) \cdot\left\{\left(2^{a_{1}}-a_{1}-1\right)\left(\begin{array}{c}
a_{2}-1 \\
c_{2}
\end{array}\right)\left(\begin{array}{c}
b_{2} \\
c_{2}
\end{array}\right) y_{3} \cdots y_{s}+0\right\} \\
&+\left(\begin{array}{c}
b_{1}-1 \\
c_{1}+1-1
\end{array}\right)\left(\begin{array}{c}
a_{2}-1 \\
c_{2}
\end{array}\right)\left(\begin{array}{c}
b_{2} \\
c_{2}
\end{array}\right) y_{3} \cdots y_{s} \\
&=\left(2^{a_{1}}-a_{1}-1\right)\left(\begin{array}{c}
b_{1} \\
c_{1}
\end{array}\right) y_{2} \cdots y_{s}+\left(\begin{array}{c}
b_{1}-1 \\
c_{1}
\end{array}\right) y_{2} \cdots y_{s} .
\end{aligned}
$$

For example, for $n=5$ with the convention $\bar{k}=-k$, we can calculate

$$
\left(\mu_{X}, \tau_{\{\overline{1}\}}^{2} \tau_{\{\overline{1}, 3,4,5, \overline{2}\}^{3}}\right)=-4
$$

by the case (ii-b) of Theorem 18 (See Figure 8). 


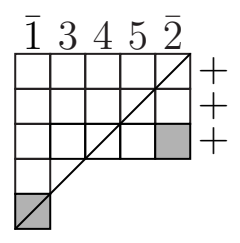

Figure 8: The Young diagram corresponding to $\tau_{\{\overline{1}\}^{2}} \tau_{\{\overline{1}, 3,4,5, \overline{2}\}}{ }^{3}$

For each even signed permutation $u \in \widetilde{\mathfrak{S}}_{n}^{+}$, an element $i \in[n]$ satisfies $u\left(\alpha_{i}\right) \in \Phi^{-}$if and only if

(D-1) if $i \leqslant n-2$, then $u(i)>u(i+1)$ with the same sign, or $u(i)<u(i+1)$ with different signs,

(D-2) if $i=n-1$, then $u(n-1), u(n)<0$, or $u(n-1)$ and $u(n)$ have different signs and the absolute value of the negative one is less than the positive one,

(D-3) if $i=n$, then $u(n-1)>u(n)$ with the same sign, or $u(n-1)<u(n)$ with different signs.

Consider the similar condition

(A-1) if $i \leqslant n-2$, then $u(i)<u(i+1)$ with the same sign or $u(i)>u(i+1)$ with different signs,

(A-2) if $i=n-1$, then $u(n-1), u(n)>0$, or $u(n-1)$ and $u(n)$ have different signs and the absolute value of the negative one is greater than the positive one,

(A-3) if $i \neq n$, then $u(n-1)<u(n)$ with the same sign or $u(n-1)>u(n)$ with different signs,

Denote

$$
\begin{aligned}
& D(u):=\{u[i] \mid i \leqslant n-2 \text { and } i \text { satisfies (D) }\} \\
& \cup\left\{u[n]_{+} \mid i=n-1 \text { satisfies (D) }\right\} \cup\left\{u[n]_{-} \mid i=n \text { satisfies (D) }\right\} \\
& \begin{aligned}
A(u):=\{u[i] \mid & i \text { satisfies }(\mathrm{A})\} \\
& \cup\left\{u[n]_{+} \mid i=n-1 \text { satisfies (A) }\right\} \cup\left\{u[n]_{-} \mid i=n \text { satisfies (A) }\right\}
\end{aligned}
\end{aligned}
$$

where

$$
[n]_{+}=\{1,2, \cdots, n-1, n\} \quad \text { and } \quad[n]_{-}=\{1,2, \cdots, n-1,-n\}
$$

(cf. Figure 7). We define a signed Young diagram $\lambda_{u, v}^{w}$ for $u, v, w \in \widetilde{\mathfrak{S}}_{n}^{+}$in the manner described in Section 4. Note that we put $I(\emptyset)=0$ as a convention.

Now, the intersection number of $Y^{w}, X_{u}$ and $X_{v}$ in $X$ of type $D_{n}$ is given by the following. 
Corollary 20. For even signed permutations $u, v, w \in \widetilde{\mathfrak{S}}_{n}^{+}$, we have

$$
\left(\mu_{X},\left[Y^{w}\right]\left[X_{u}\right]\left[X_{v}\right]\right)=\widetilde{I}\left(\lambda_{u v}^{w}\right)
$$

where $\widetilde{I}=(-1)^{n+s} \widetilde{y}_{1} y_{2} \cdots y_{s}$ is the function described in Theorem 18 .

For example, for $n=5$ with the convention $\bar{k}=-k$, the Young diagram $\lambda_{\overline{1} 345 \overline{2}, \overline{1} \overline{1} 345 \overline{2}}$ is the one in Figure 8, and hence we obtain

$$
\left(\mu_{X},\left[Y^{\overline{1} \overline{2} 543}\right]\left[X_{\overline{1} 345 \overline{2}}\right]\left[X_{\overline{1} 345 \overline{2}}\right]\right)=-4 .
$$

\section{$6 \quad$ On exceptional types}

In this section, we include the computation of intersection numbers of invariant divisors of the toric manifold $X$ for the root system of exceptional type $G_{2}$. For other exceptional types $F_{4}, E_{6}, E_{7}$, and $E_{8}$, it would be interesting to find combinatorial objects which effectively compute the intersection numbers of invariant divisors.

Let $E=\left\{x \in \mathbb{R}^{3} \mid x_{1}+x_{2}+x_{3}=0\right\}$. The roots are

$$
\begin{aligned}
& \pm\left(t_{1}-t_{2}\right), \pm\left(t_{1}-t_{3}\right), \pm\left(t_{2}-t_{3}\right), \\
& \pm\left(2 t_{1}-t_{2}-t_{3}\right), \pm\left(2 t_{2}-t_{1}-t_{3}\right), \pm\left(2 t_{3}-t_{1}-t_{2}\right)
\end{aligned}
$$

where $t_{i} \in \mathbb{R}^{3}$ is the $i$-th standard vector. We choose $\Pi=\left\{t_{1}-t_{2},-2 t_{1}+t_{2}+t_{3}\right\}$ as the set of simple roots, and write $\alpha_{1}=t_{1}-t_{2}$ and $\alpha_{2}=-2 t_{1}+t_{2}+t_{3}$. The Weyl group $W$ is the dihedral group of order 12 which is identified with the subgroup

$$
W_{G_{2}}:=\left\{u \in \widetilde{\mathfrak{S}}_{3} \mid u(1), u(2), \text { and } u(3) \text { have the same sign }\right\}
$$

of the 3rd signed permutation group. Under this identification, the action of the Weyl group on $E$ is written as the natural action of $W_{G_{2}}$ on the indexes $i$ of $t_{i} ; u \cdot t=t_{u(1)}$ for $u \in W_{G_{2}}$ where $t_{-i}:=-t_{i}(1 \leqslant i \leqslant 3)$. This action of $W_{G_{2}}$ preserves $\Phi$. The minimal generators $\omega_{1}, \omega_{2} \in E^{*}$ of the fundamental Weyl chamber $\sigma_{\text {id }}$ are

$$
\omega_{1}=e_{3}-e_{2}, \omega_{2}=\frac{1}{3}\left(2 e_{3}-e_{1}-e_{2}\right)
$$

where $\left\{e_{i}\right\}_{i} \subset\left(\mathbb{R}^{3}\right)^{*}$ is the dual basis of $\left\{t_{i}\right\}_{i} \subset \mathbb{R}^{3}$.

Denoting by $2^{[ \pm 3]}$ the set of all subsets of $[ \pm 3]=\{1,2,3,-1,-2,-3\}$, we have a well-defined map $\Phi^{*} \rightarrow 2^{[n+1]}$ by sending

$$
e_{u(3)}-e_{u(2)} \mapsto\{u(3),-u(2)\}, \quad \frac{1}{3}\left(2 e_{u(3)}-e_{u(1)}-e_{u(2)}\right) \mapsto\{u(3)\}
$$

for $u \in W_{G_{2}}$. This is an injection, and hence we can identify $\Phi^{*}$ with the following subset of $2^{[ \pm 3]}$;

$$
\mathcal{S}:=\{3 \overline{2}, \overline{3} 2,3 \overline{1}, \overline{3} 1,2 \overline{1}, \overline{2} 1,3, \overline{3}, 2, \overline{2}, 1, \overline{1}\}
$$


where $\bar{k}=-k$ for $1 \leqslant k \leqslant 3$ and each sequence $a b$ in $\mathcal{S}$ is the set $\{a, b\}$, i.e. $3 \overline{2}=\{3,-2\}$ for example. Now, for each $S \in \mathcal{S}$, we have $\tau_{S}:=\tau_{u \omega_{i}} \in H^{2}(X)$ where $u \omega_{i} \in \Phi^{*}$ corresponds to $S$ by this identification. Then, for $S_{1}, S_{2} \in \mathcal{S}$, it follows by Lemma 2 that $\tau_{S_{1}} \tau_{S_{2}}=0$ unless these sets form a nested chain of subsets, i.e. $S_{1} \subset S_{2}$ or $S_{1} \supset S_{2}$.

The linear relations (3) for $\alpha=\alpha_{1}, \alpha_{2}$ are translated to

$$
\begin{aligned}
& \tau_{3 \overline{2}}+\tau_{\overline{3} 1}+2 \tau_{\overline{2} 1}+\tau_{\overline{2}}+\tau_{1}=\tau_{\overline{3} 2}+\tau_{3 \overline{1}}+2 \tau_{2 \overline{1}}+\tau_{2}+\tau_{\overline{1}}, \\
& 3 \tau_{3 \overline{1}}+3 \tau_{2 \overline{1}}+\tau_{3}+\tau_{2}+2 \tau_{\overline{1}}=3 \tau_{\overline{3} 1}+3 \tau_{\overline{2} 1}+\tau_{\overline{3}}+\tau_{\overline{2}}+2 \tau_{1},
\end{aligned}
$$

respectively. From these relations together with the above observation about the vanishing of $\tau_{S_{1}} \tau_{S_{2}}$, we see that

$$
\tau_{3 \overline{2}} \tau_{3}=1, \tau_{3 \overline{2}} \tau_{3 \overline{2}}=-1, \tau_{3} \tau_{3}=-3
$$

Now, let

$$
I_{G_{2}}(2,1):=1, I_{G_{2}}(1,1):=-3, I_{G_{2}}(2,2):=-1
$$

where $(2,1),(1,1)$, and $(2,2)$ are Young diagrams with 2 rows. Now the next claim follows from Lemma 3 ; if $S_{1}, S_{2} \in \mathcal{S}$ form a nested chain of subsets, then we have

$$
\left(\mu_{X}, \tau_{S_{1}} \tau_{S_{2}}\right)=I_{G_{2}}(\lambda)
$$

where $\mu_{X}$ is the fundamental homology class and $\lambda$ is the Young diagram consisting of $\left|S_{1}\right|$ and $\left|S_{2}\right|$ reordered as a weakly decreasing sequence. Otherwise, the intersection number is zero.

Finally, we list the presentations of $\left[X_{u}\right]$ as monomials of $\tau_{S}$ for all $u \in W_{G_{2}}$ in one-line notations;

$$
\begin{array}{ll}
{\left[X_{123}\right]=1, \quad\left[X_{213}\right]=\tau_{3 \overline{1}},\left[X_{132}\right]=\tau_{2 \overline{3}},\left[X_{231}\right]=\tau_{1 \overline{3}},\left[X_{312}\right]=\tau_{2}, \quad\left[X_{321}\right]=\tau_{1},} \\
{\left[X_{\overline{1} \overline{2} \overline{3}]}=\tau_{\overline{3} 2} \tau_{\overline{3}}, \quad\left[X_{\overline{2} \overline{1} \overline{3}]}\right]=\tau_{\overline{3}},\left[X_{\overline{1} \overline{3} \overline{2}]}\right]=\tau_{\overline{2}}, \quad\left[X_{\overline{2} \overline{3} \overline{1}}\right]=\tau_{\overline{1}}, \quad\left[X_{\overline{3} \overline{1} \overline{2}]}\right]=\tau_{\overline{2} 1}, \quad\left[X_{\overline{3} \overline{2} \overline{1}}\right]=\tau_{\overline{1} 2} .\right.}
\end{array}
$$

Since we have $\left[Y^{u}\right]=\left(w_{0}^{-1}\right)^{*}\left[X_{w_{0} u}\right]=w_{0}^{*}\left[X_{w_{0} u}\right]$ where $w_{0}=\overline{1} \overline{2} \overline{3}$ is the longest permutation, we obtain the list of $\left[Y^{u}\right]$;

$$
\begin{array}{ll}
{\left[Y^{\overline{1} \overline{2} \overline{3}}\right]=1,} & {\left[Y^{\overline{2} \overline{1} \overline{3}}\right]=\tau_{\overline{3} 1},\left[Y^{\overline{1} \overline{3} \overline{2}}\right]=\tau_{\overline{2} 3},\left[Y^{\overline{2} \overline{3} \overline{1}}\right]=\tau_{\overline{1} 3},\left[Y^{\overline{3} \overline{1} \overline{2}}\right]=\tau_{\overline{2}}, \quad\left[Y^{\overline{3} \overline{2} \overline{1}}\right]=\tau_{\overline{1}},} \\
{\left[Y^{123}\right]=\tau_{3 \overline{2}} \tau_{3}, \quad\left[Y^{213}\right]=\tau_{3},\left[Y^{132}\right]=\tau_{2},\left[Y^{231}\right]=\tau_{1},\left[Y^{312}\right]=\tau_{2 \overline{1}}, \quad\left[Y^{321}\right]=\tau_{1 \overline{2}} .}
\end{array}
$$

With these lists, we can compute intersection numbers $\left(\mu_{X},\left[Y^{w}\right]\left[X_{u}\right]\left[X_{v}\right]\right)$ for all $u, v, w \in$ $W_{G_{2}}$ by $(25)$.

\section{Acknowledgements}

The author would like to thank Tatsuya Horiguchi, Hiroaki Ishida, Ivan Limonchenko, and Tomoo Matsumura for valuable comments. He also thanks Miho Hatanaka for reading of the first version of this paper carefully. 


\section{References}

[1] V. Batyrev and Mark Blume, The functor for toric varieties associated with Weyl chambers and Losev-Manin moduli spaces, Tohoku Math. J. 63 (2011), 581-604.

[2] A. Björner and F. Brenti, Combinatorics of Coxeter groups, Graduate Texts in Mathematics 231, Springer, New York, 2005.

[3] D. Cox, J. Little and H. Schenck, Toric varieties, Graduate Studies in Mathematics, 124, American Mathematical Society, Providence RI (2011).

[4] F. De Mari, C. Procesi and M. A. Shayman, Hessenberg varieties, Trans. Amer. Math. Soc. 332 (1992), no. 2, 529-534.

[5] V. Dolgachev and V. Lunts, A character formula for the representation of a Weyl group in the cohomology of the associated toric variety, J. Algebra 168 (1994), 741772 .

[6] W. Fulton, An Introduction to Toric Varieties, Ann. of Math. Stud., vol. 113, Princeton Univ. Press, Princeton NJ, 1993.

[7] A. Klyachko, Orbits of a maximal torus on a flag space, Functional Anal. Appl. 19 (1985), no. 2, 65-66.

[8] A. Losev and Y. Manin, New moduli spaces of pointed curves and pencils of flat connections, Michigan Math. J. 48 (2000), 443-472.

[9] C. Procesi, The toric variety associated to Weyl chambers, Mots, 153-161, Lang. Raison. Calc., Hermès, Paris, 1990.

[10] J. Stembridge, Some permutation representations of Weyl groups associated with the cohomology of toric varieties, Adv. Math. 106 (1994), 244-301. 